\title{
A PATH ANALYSIS OF SOME COMMUNITY FACTORS AFFECTING ON POLITICAL PARTICIPATION OF THE INHABITANTS OF RURAL COMMUNITIES IN SHARKIA GOVERNORATE-EGYPT
}

Salama, F. A.* and A. A. M. H. Ecresh**

* Faculty of Agriculture - Minufiya University

${ }^{* *}$ Faculty of Agriculture - Zagazig University

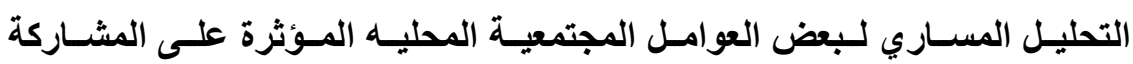

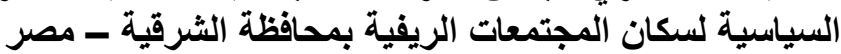

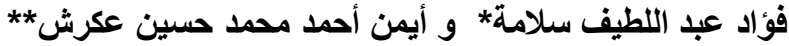

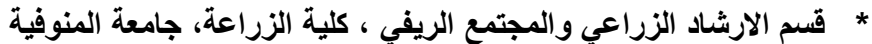

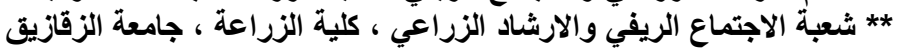

الملخص المص

استهدف هذه الدر اسة التعرف على أهم العو امل المجتمعية المؤثرة على مستوى المشاركة السياسية

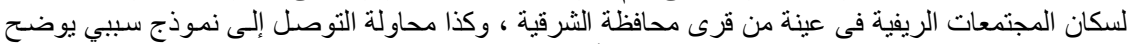

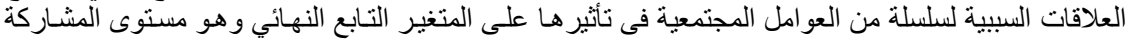

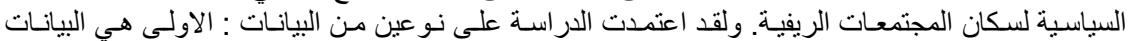

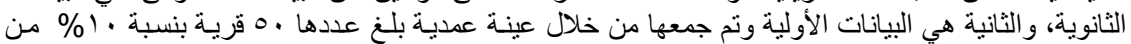

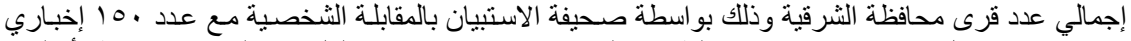

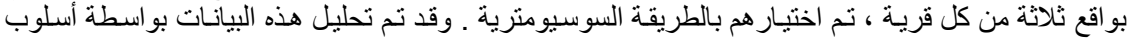

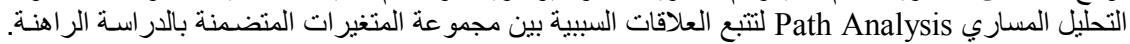

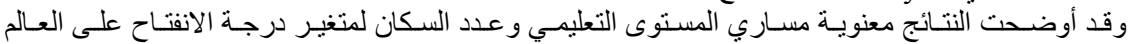

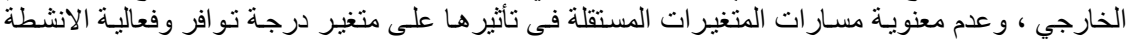

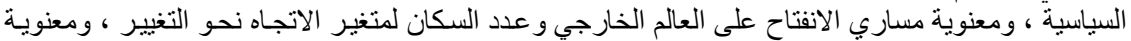

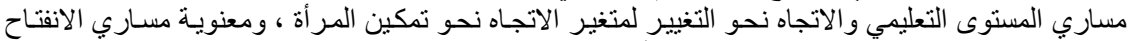

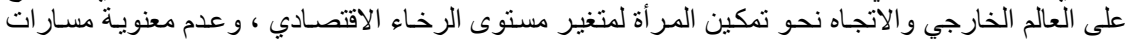

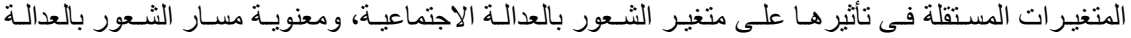

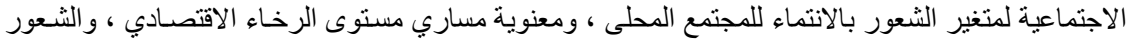

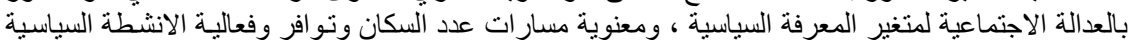

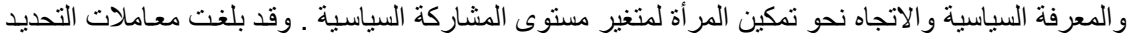

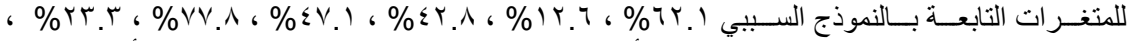

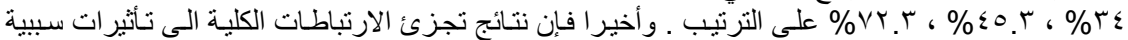

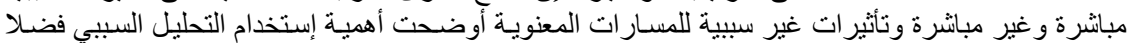

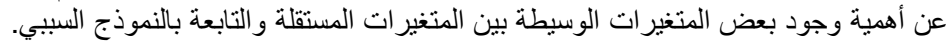

المقدمة - 2 المبرة

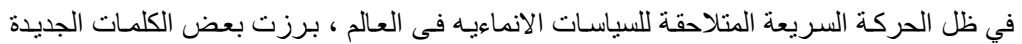

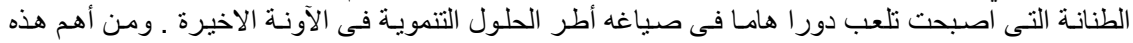

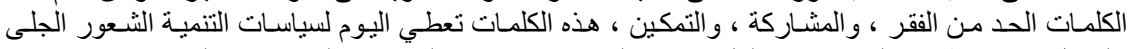

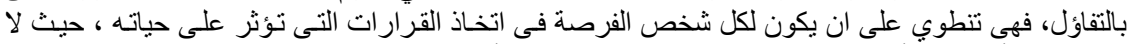

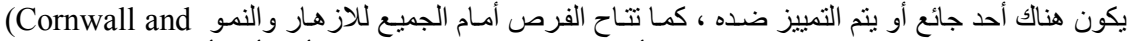
. Brock , 2005, ppiii-1i)

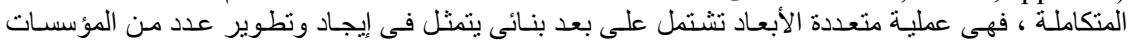

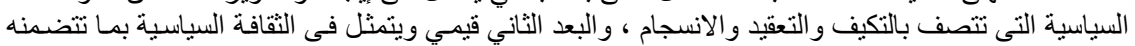




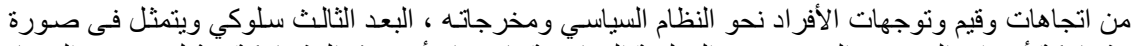

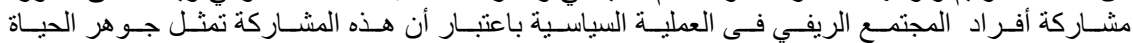

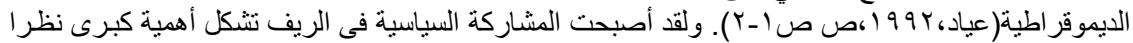

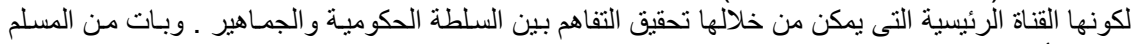

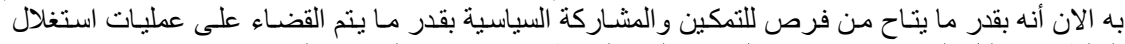

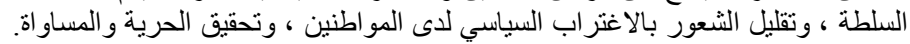

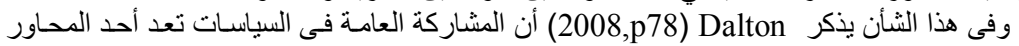

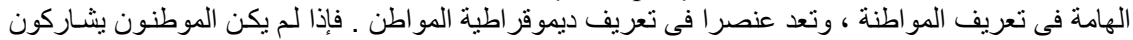

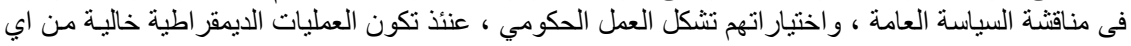

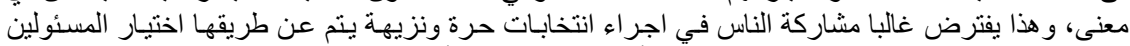

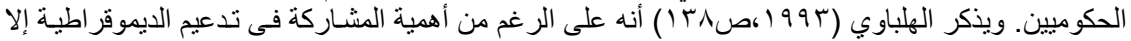

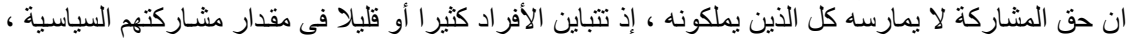

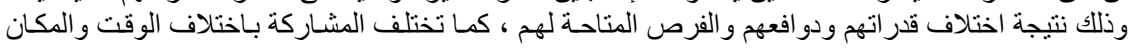

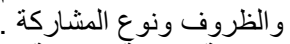
المشكلة البحثيةٌ للار استة:

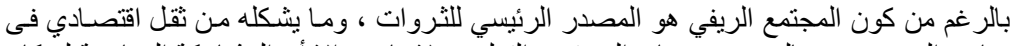

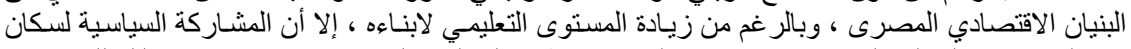

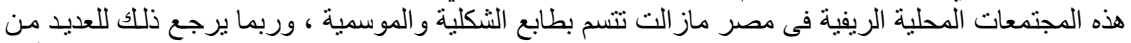

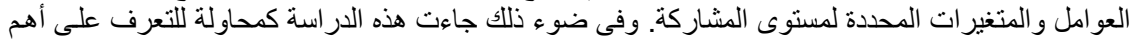

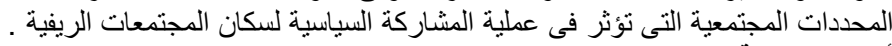

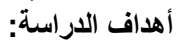

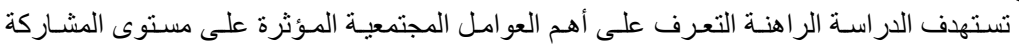

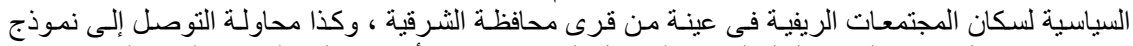

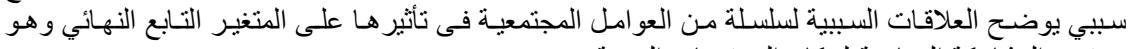

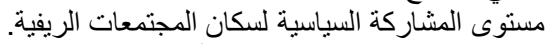

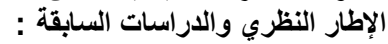

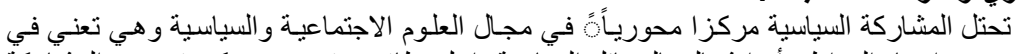

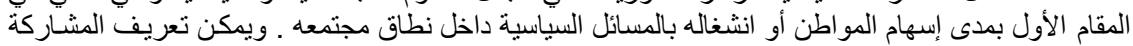

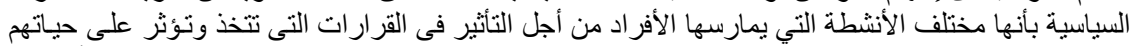

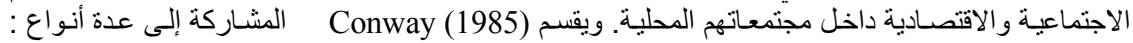

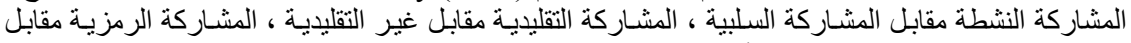

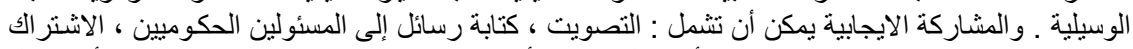

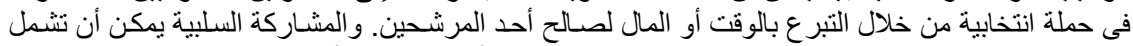

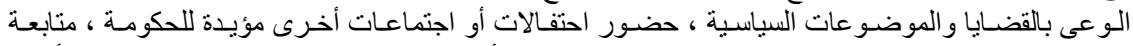

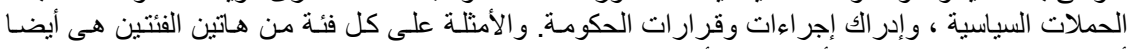

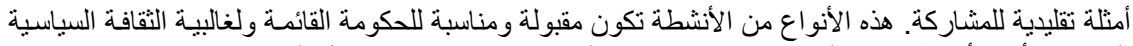

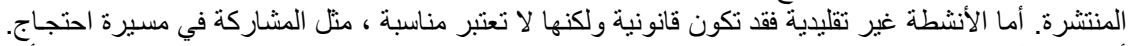

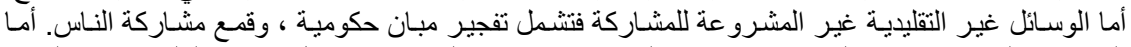

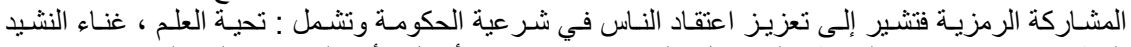

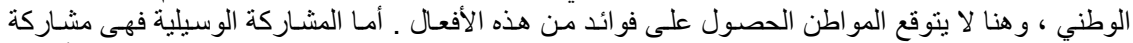

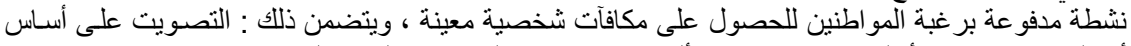

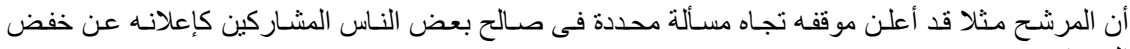

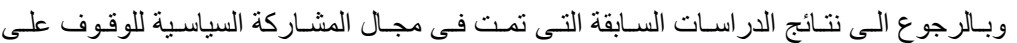
الضر ائب البرن

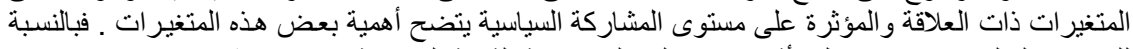

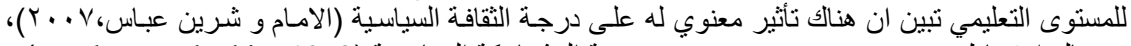

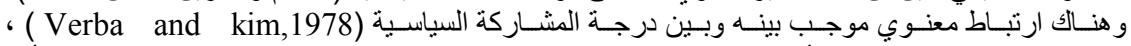
(Pindare,1984) 


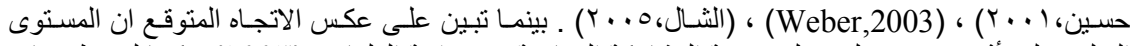

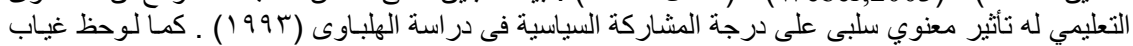

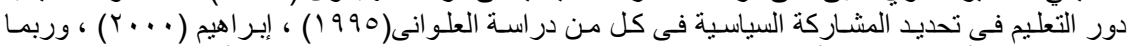

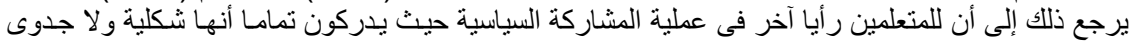

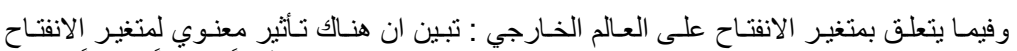

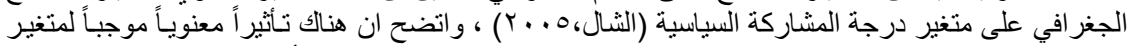

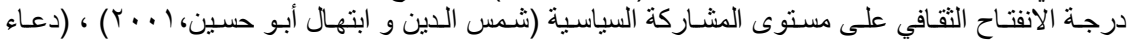

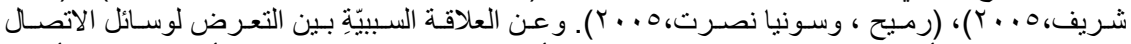

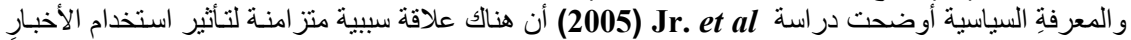

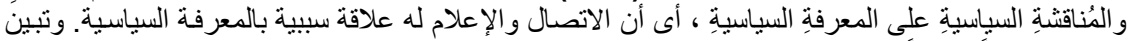

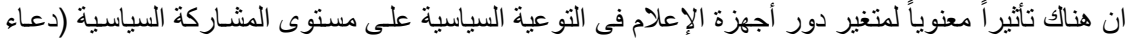

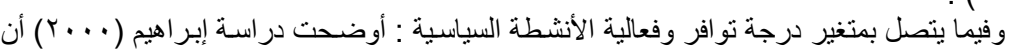

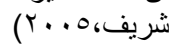

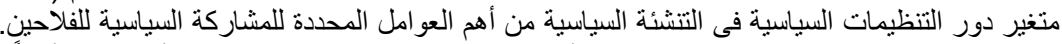

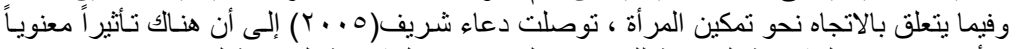

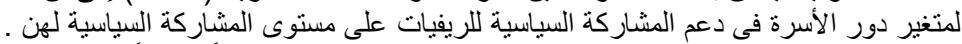

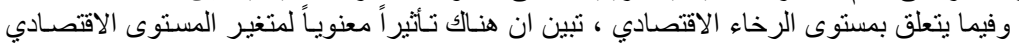

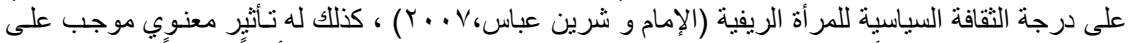

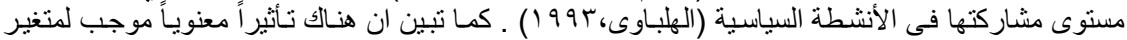

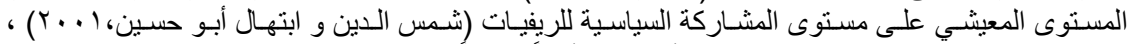

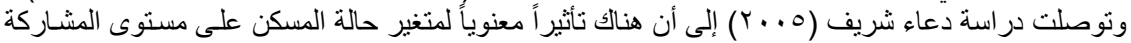

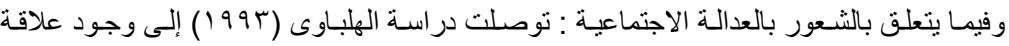

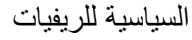

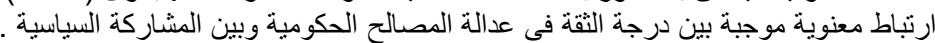

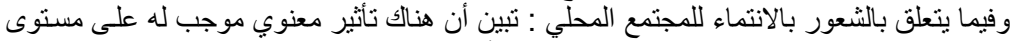

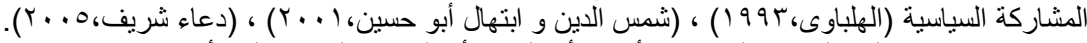

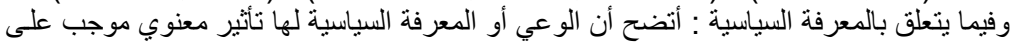

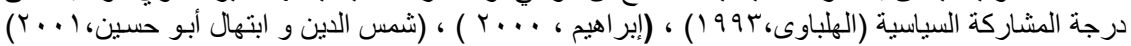

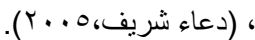

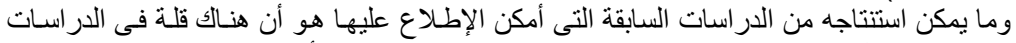

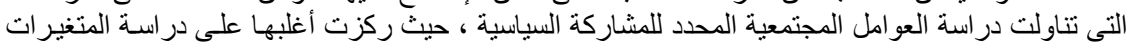

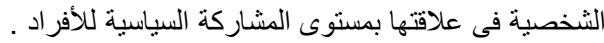

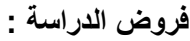

تتبنى الدر اسـة الر اهنـة نموذجـا سببيا مقترحسا للمشـاركة السياسية لسكان المجتمعـات الريفيـة بستند على الفروض البحثية النظرية التالية : النية ا-توجد علاقة معنوية بين المتغيرين التاليين مجتمعين : عدد السكان ، المستوى التعليمي، وبين متغير درجة الانفتاح على العالم الخارجي.

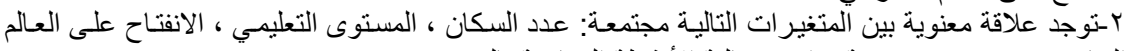

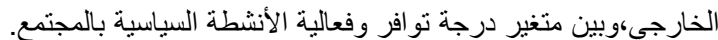

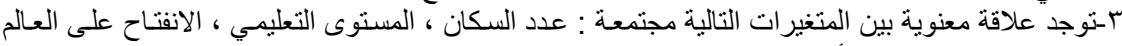

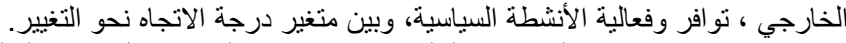

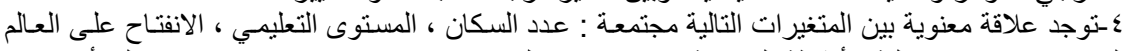

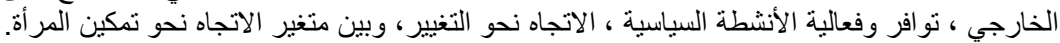

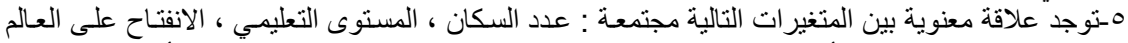

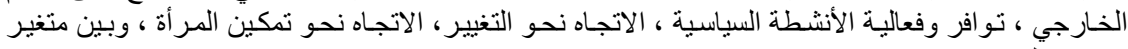
مستوى ألرخاء الاقتصادي. 


\section{J. Agric. Sci. Mansoura Univ., 33(11), November, 2008}

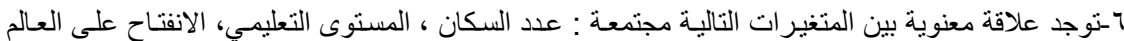

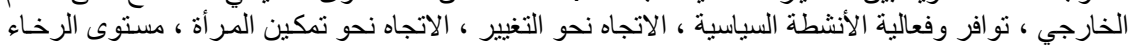

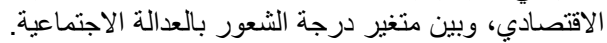

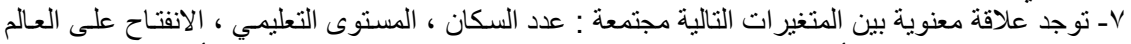

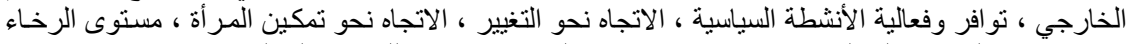

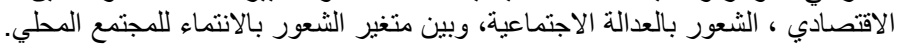

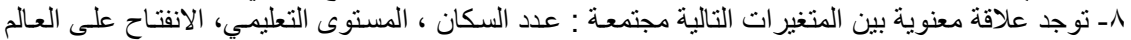

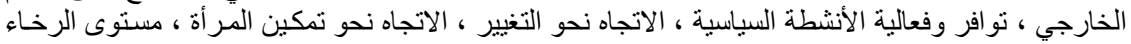

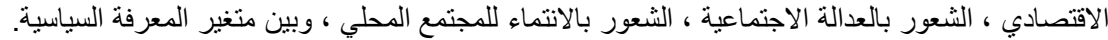

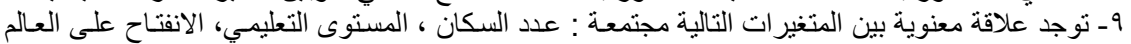

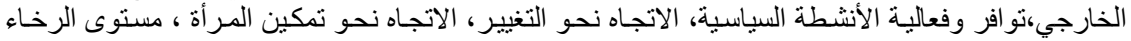

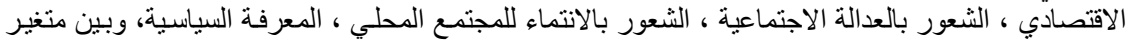

مستوى المشاركة السياسية.

\section{منهجية الدراسة وأدواتها}

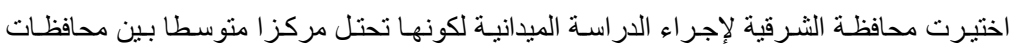

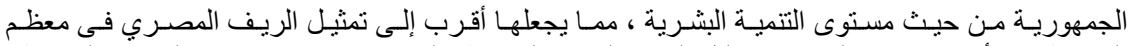

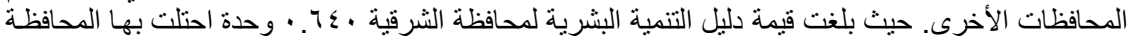

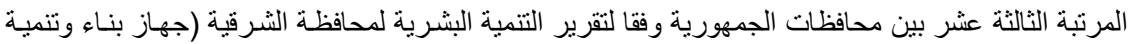

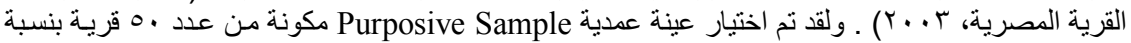

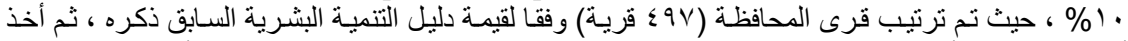

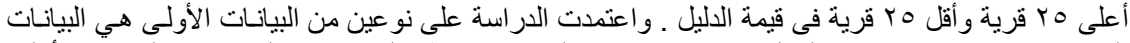

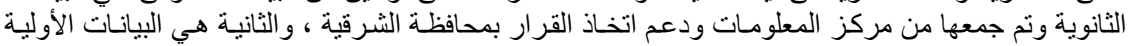

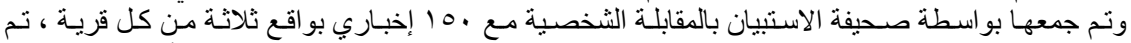

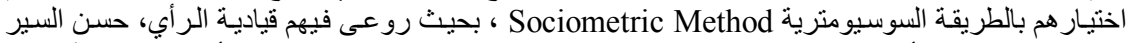

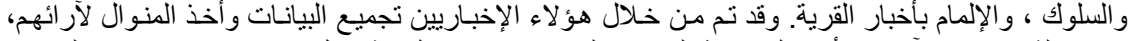

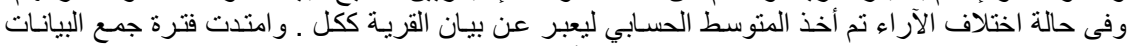

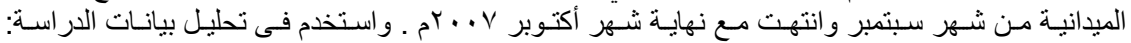

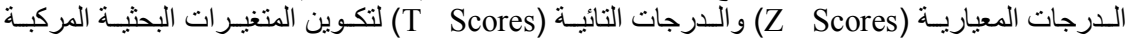

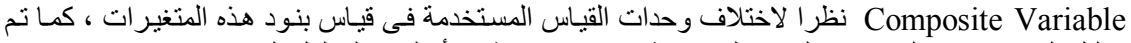

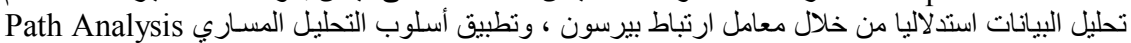

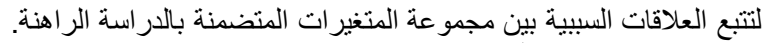

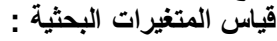

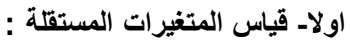

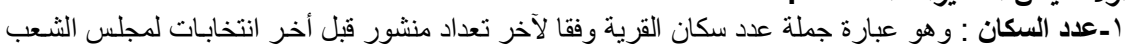

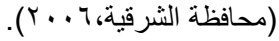

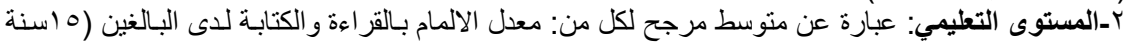

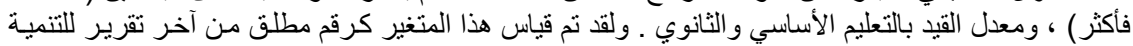

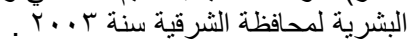

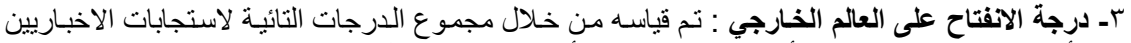

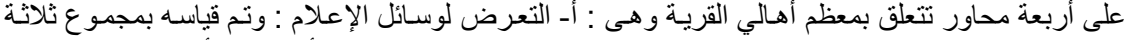

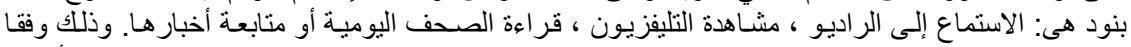

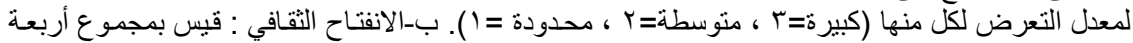

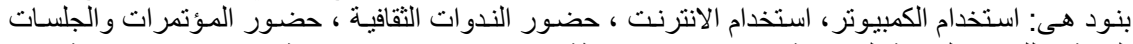

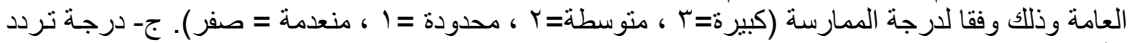

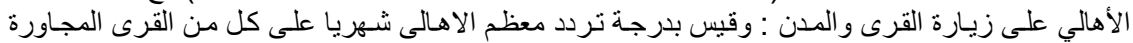

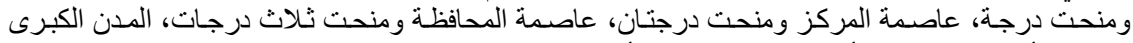

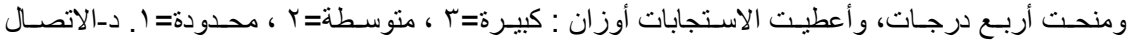

$\Lambda \mathrm{\wedge}$. 


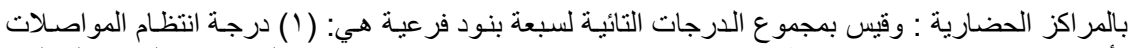

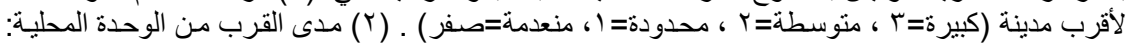

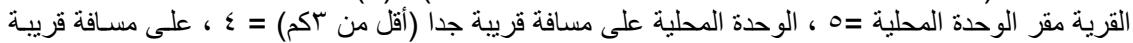

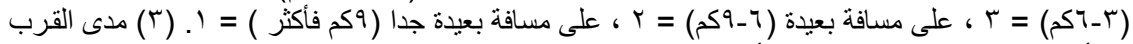

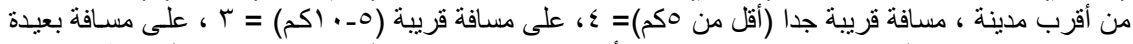

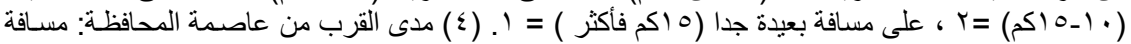

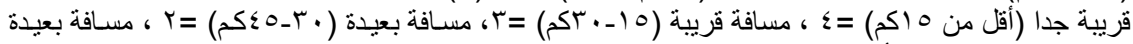

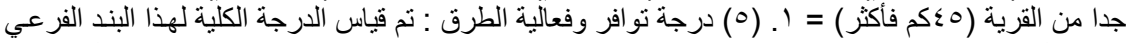

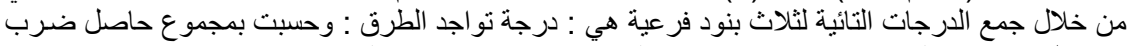

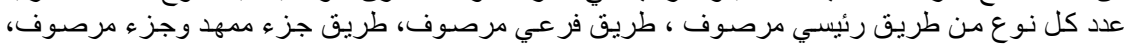

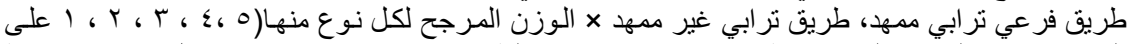

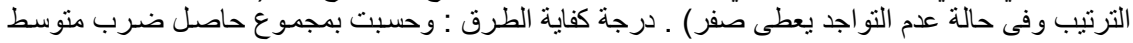

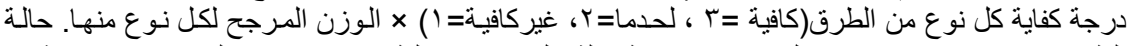

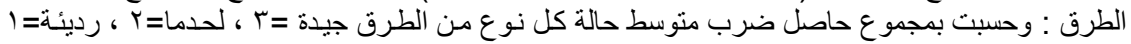

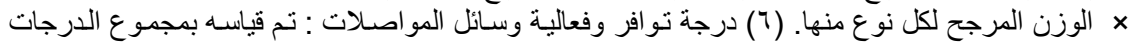

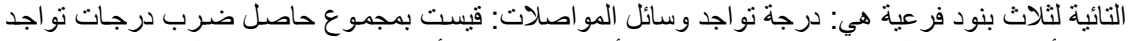

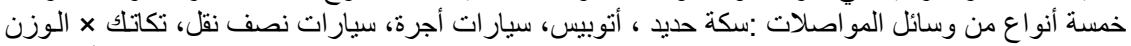

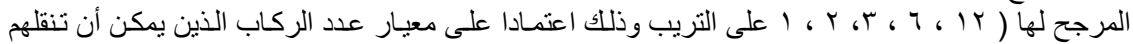

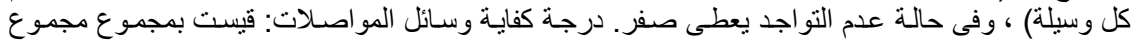

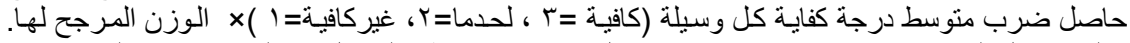

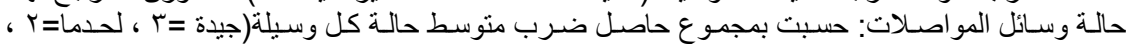

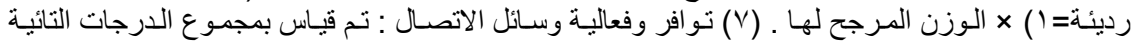

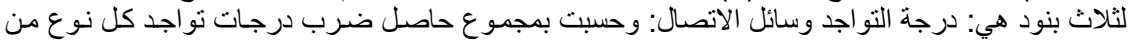

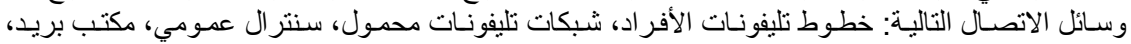

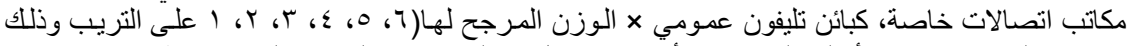

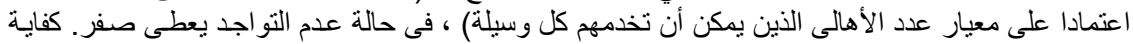

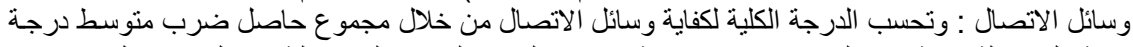

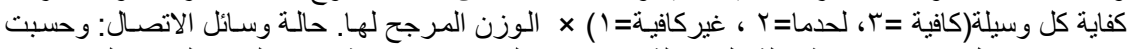

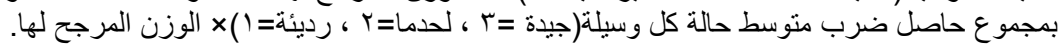

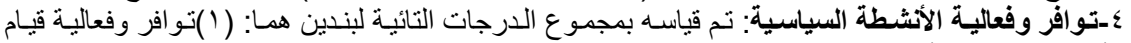

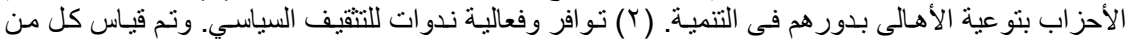

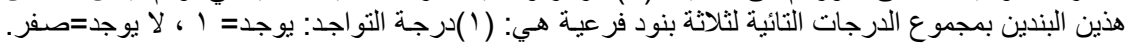

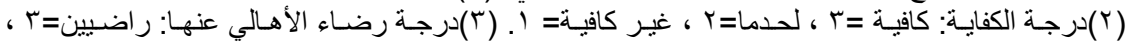

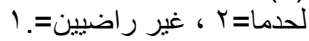

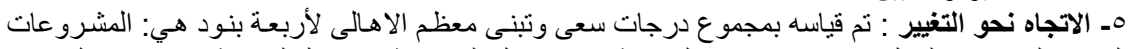

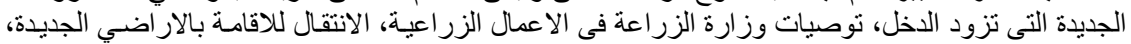

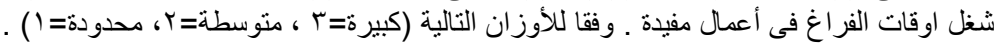

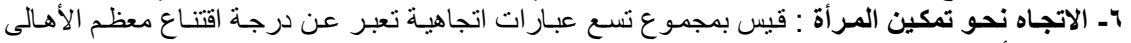

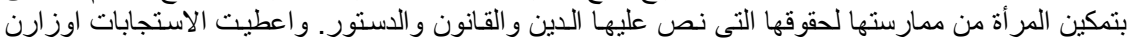

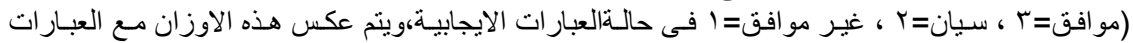

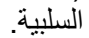

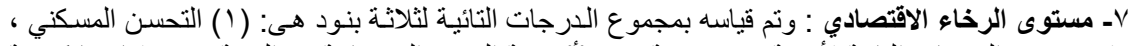

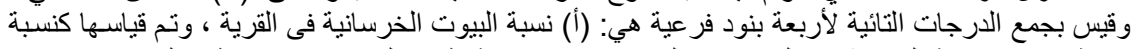

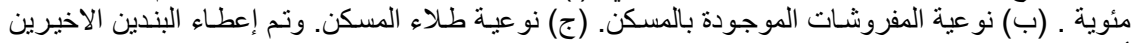

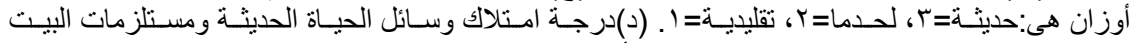

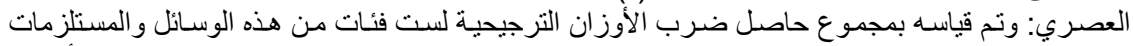

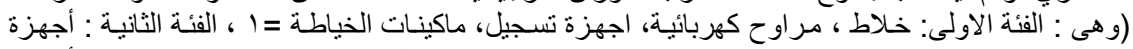

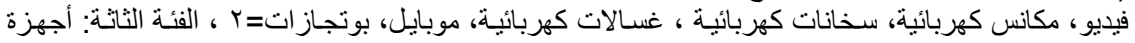




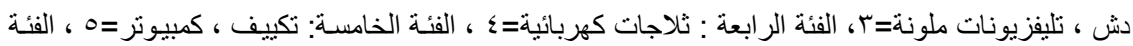

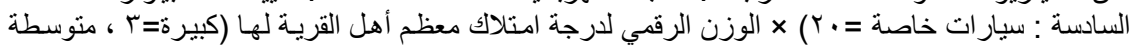

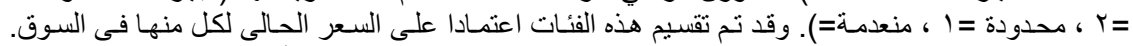

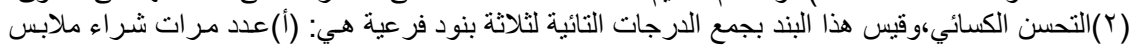

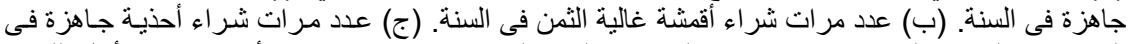

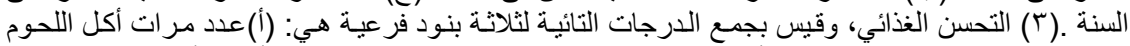

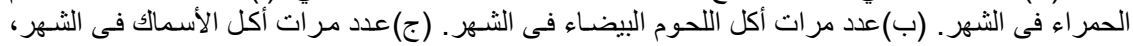

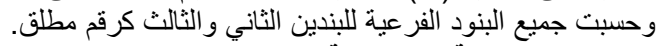

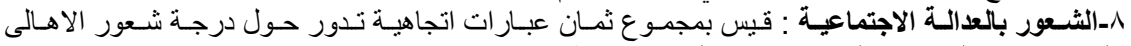

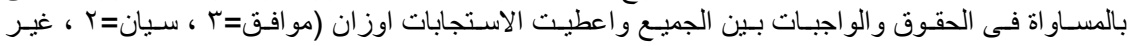

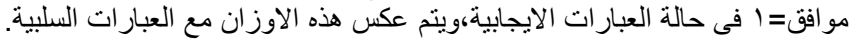

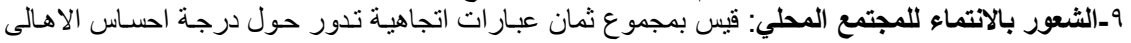

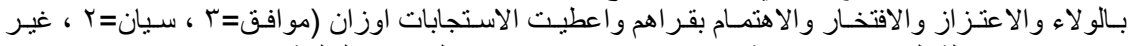

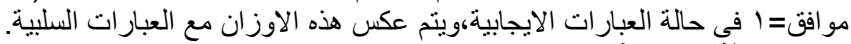

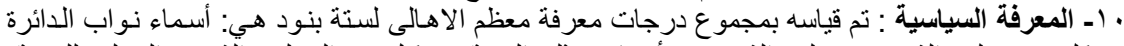

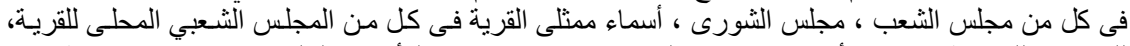

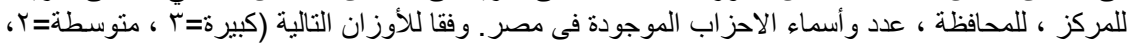

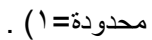

ثانيا- قياس المتغير التابع الرئيسي للاراسـة ، وهو مستوى المشاركة السياسية لسكان المجتمعات الريفية:

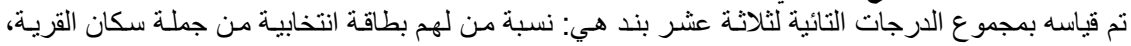

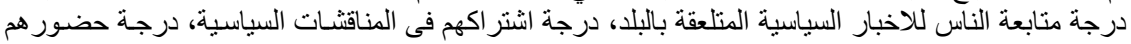

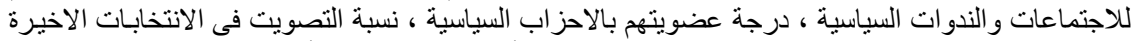

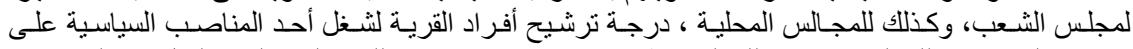

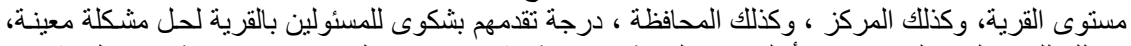

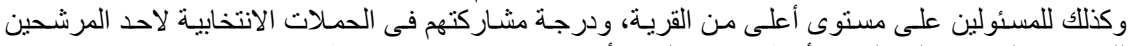

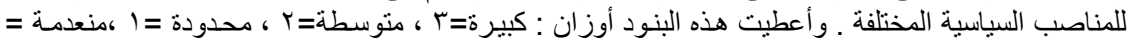

ويتضـمن النمـوذج السببي المقترح في هذه الدراسـة لمستوى المشـاركة السياسية لسكان المجتمعـات صفر.

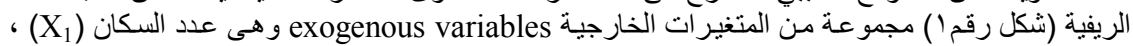

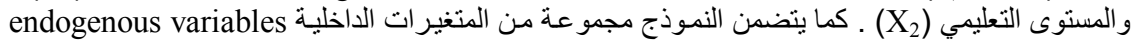

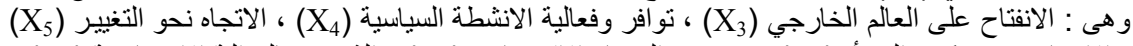

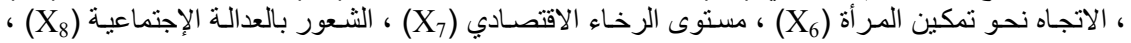

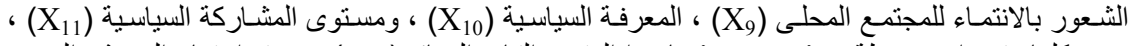

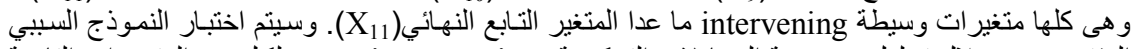

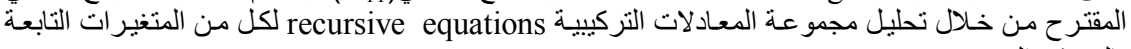

$\mathrm{X}_{3}=\alpha+\mathrm{P}_{31} \mathrm{X}_{1}+\mathrm{P}_{32} \mathrm{X}_{2}+\mathrm{P}_{30} \mathrm{R}_{\mathrm{o}}$

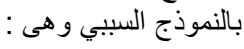

$\mathrm{X}_{4}=\alpha+\mathrm{P}_{41} \mathrm{X}_{1}+\mathrm{P}_{42} \mathrm{X}_{2}+\mathrm{P}_{43} \mathrm{X}_{3}+\mathrm{P}_{4 \mathrm{q}} \mathrm{R}_{\mathrm{q}}$

$\mathrm{X}_{5}=\alpha+\mathrm{P}_{51} \mathrm{X}_{1}+\mathrm{P}_{52} \mathrm{X}_{2}+\mathrm{P}_{53} \mathrm{X}_{3}+\mathrm{P}_{54} \mathrm{X}_{4}+\mathrm{P}_{5 \mathrm{~s}} \mathrm{R}_{\mathrm{s}}$

$\mathrm{X}_{6}=\alpha+\mathrm{P}_{61} \mathrm{X}_{1}+\mathrm{P}_{62} \mathrm{X}_{2}+\mathrm{P}_{63} \mathrm{X}_{3}+\mathrm{P}_{64} \mathrm{X}_{4}+\mathrm{P}_{65} \mathrm{X}_{5}+\mathrm{P}_{6 \mathrm{u}} \mathrm{R}_{\mathrm{u}}$

$\mathrm{X}_{7}=\alpha+\mathrm{P}_{71} \mathrm{X}_{1}+\mathrm{P}_{72} \mathrm{X}_{2}+\mathrm{P}_{73} \mathrm{X}_{3}+\mathrm{P}_{74} \mathrm{X}_{4}+\mathrm{P}_{75} \mathrm{X}_{5}+\mathrm{P}_{76} \mathrm{X}_{6}+\mathrm{P}_{7 \mathrm{v}} \mathrm{R}_{\mathrm{v}}$

$\mathrm{X}_{8}=\alpha+\mathrm{P}_{81} \mathrm{X}_{1}+\mathrm{P}_{82} \mathrm{X}_{2}+\mathrm{P}_{83} \mathrm{X}_{3}+\mathrm{P}_{84} \mathrm{X}_{4}+\mathrm{P}_{85} \mathrm{X}_{5}+\mathrm{P}_{86} \mathrm{X}_{6}+\mathrm{P}_{87} \mathrm{X}_{7}+\mathrm{P}_{8 \mathrm{v}} \mathrm{R}_{\mathrm{x}}$

$\mathrm{X}_{9}=\alpha+\mathrm{P}_{91} \mathrm{X}_{1}+\mathrm{P}_{92} \mathrm{X}_{2}+\mathrm{P}_{93} \mathrm{X}_{3}+\mathrm{P}_{94} \mathrm{X}_{4}+\mathrm{P}_{95} \mathrm{X}_{5}+\mathrm{P}_{96} \mathrm{X}_{6}+\mathrm{P}_{97} \mathrm{X}_{7}+\mathrm{P}_{98} \mathrm{X}_{8}+\mathrm{P}_{9 \mathrm{w}}$ $\mathrm{R}_{\mathrm{w}}$

$\mathrm{X}_{10}=\alpha+\mathrm{P}_{101} \mathrm{X}_{1}+\mathrm{P}_{102} \mathrm{X}_{2}+\mathrm{P}_{103} \mathrm{X}_{3}+\mathrm{P}_{104} \mathrm{X}_{4}+\mathrm{P}_{105} \mathrm{X}_{5}+\mathrm{P}_{106} \mathrm{X}_{6}+\mathrm{P}_{107} \mathrm{X}_{7}+\mathrm{P}_{108} \mathrm{X}_{8}$ $+\mathrm{P}_{109} \mathrm{X}_{9}+\mathrm{P}_{10 \mathrm{y}} \mathrm{R}_{\mathrm{y}}$

$\mathrm{X}_{11}=\alpha+\mathrm{P}_{111} \mathrm{X}_{1}+\mathrm{P}_{112} \mathrm{X}_{2}+\mathrm{P}_{113} \mathrm{X}_{3}+\mathrm{P}_{114} \mathrm{X}_{4}+\mathrm{P}_{115} \mathrm{X}_{5}+\mathrm{P}_{116} \mathrm{X}_{6}+\mathrm{P}_{117} \mathrm{X}_{7}+\mathrm{P}_{118} \mathrm{X}_{8}$ $+\mathrm{P}_{119} \mathrm{X}_{9}+\mathrm{P}_{1110} \mathrm{X}_{10}+\mathrm{P}_{11 \mathrm{z}} \mathrm{R}_{\mathrm{z}}$ 


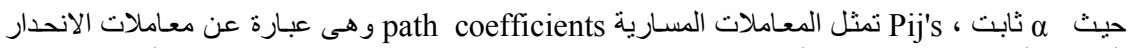

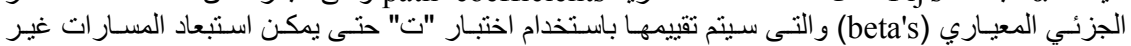

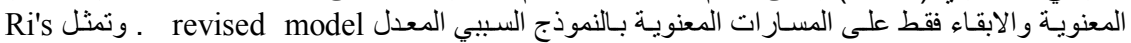

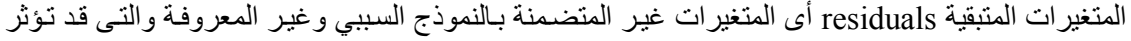

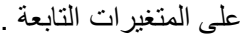

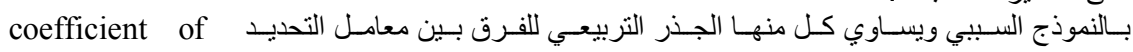
determination

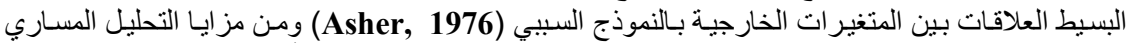

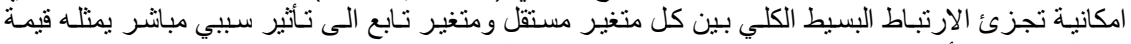

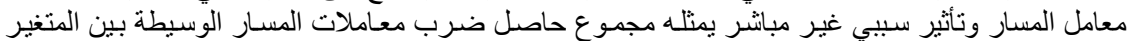

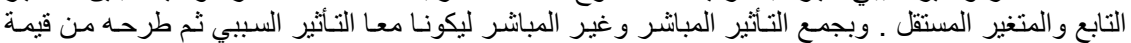

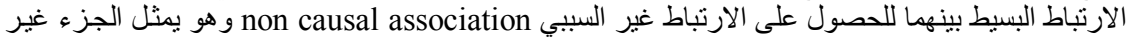

المنطقى spurious وغير المحلل بالارتباط البسيط ( Alwin and Hauser,1975 ) . و عموما نجد أن تحليل الارنباط يقسم الى : الإني :

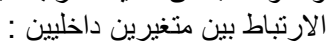

الارتباط = التاثير السببي + التأثير غير السببي + الجزء الزائف أو غير المنطقى Direct Effect + Indirect Effects + Spuriousness

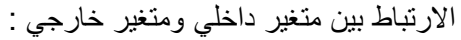
الارنباط = الناثير السببي + التأثير غير السبير + التبير

Correlation = Direct Effect + Indirect Effects + Unspecified Covariance

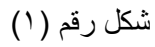




\section{نتائسج الدراسـة}

باستعر اض نتائج تحليل الانحدار المتعدد للمتغير ات التابعة بالنموذج السببي (الجدول رقم ( ) التالى لئى

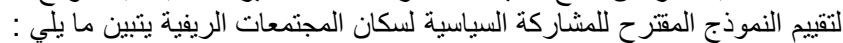

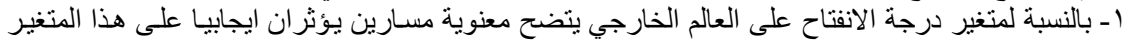

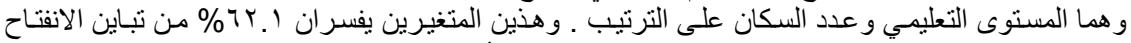

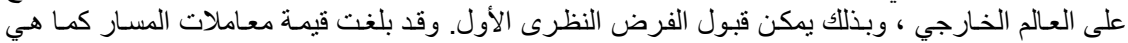

$\mathrm{X}_{3}=493.244+0.329 \mathrm{X}_{1}+0.674 \mathrm{X}_{2}+\mathrm{P}_{30} \mathrm{R}_{\mathrm{o}}$

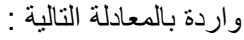

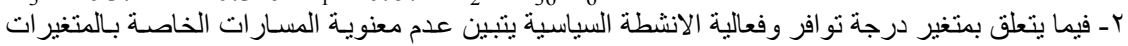

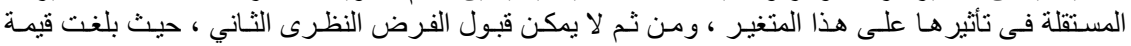
$\mathrm{X}_{4}=403.702+0.113 \mathrm{X}_{1}+0.371 \mathrm{X}_{2}-0.063 \mathrm{X}_{3}+\mathrm{P}_{4 \mathrm{q}} \mathrm{R}_{\mathrm{q}}$

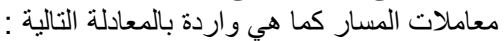

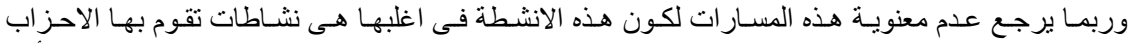

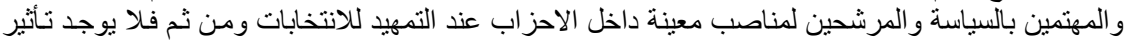

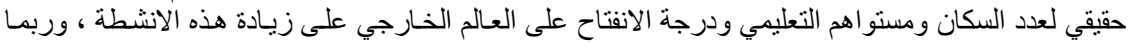

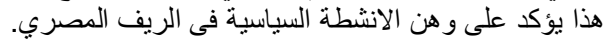

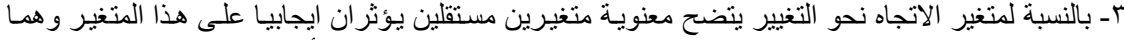

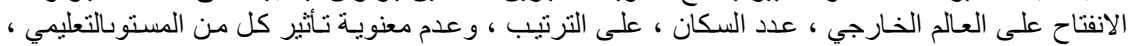

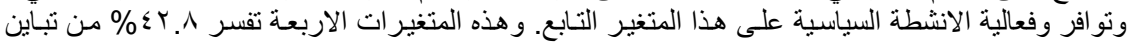

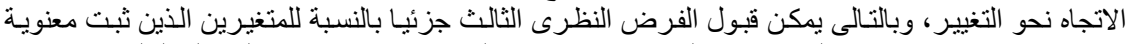

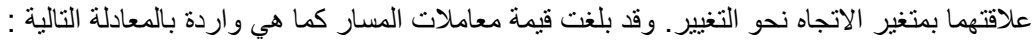

$$
\mathrm{X}_{5}=-1.941+0.382 \mathrm{X}_{1}+0.01 \mathrm{X}_{2}+0.385 \mathrm{X}_{3}+0.01 \mathrm{X}_{4}+\mathrm{P}_{5 \mathrm{~s}} \mathrm{R}_{\mathrm{s}}
$$

\begin{tabular}{|c|c|c|c|c|c|}
\hline معامل التحديد & آلمحسوبة "ته & قالمِيَة معامل & الارتباط البسيطة & |المتغيرات المستقلة & |المتغير التابع \\
\hline$\cdot .74$ & **T.7tV & $\cdot$. rरq & ${ }^{* *} \cdot .519$ & السذ & \multirow{2}{*}{ الانفتاح على العالم الخارجي } \\
\hline ف & $* * \vee . \leqslant r \Lambda$ & $.7 \vee \varepsilon$ & $* * . \vee \backslash \wedge$ & & \\
\hline$\cdot 1{ }^{\prime}$ & $\cdot 117$ & $.11 T$ & $\cdot .1 \% 7$ & & \multirow[t]{3}{*}{ تو افر وفعالِية الانثطة السياسية } \\
\hline \multirow[t]{2}{*}{ ف= } & $1 . \wedge \cdot 1$ &.$r v 1$ & *. $\cdot r \leqslant 1$ & \multirow[b]{2}{*}{ الانفتاح على التعآّم الخارجي } & \\
\hline & $. r \wedge 1-$ & $\because$ ・T- & .101 & & \\
\hline \multirow{4}{*}{ 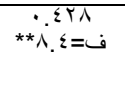 } & $* * Y .90 \leqslant$ & $\cdot$ TNT & $\star * * .0 \leq V$ & & \multirow{4}{*}{ |الاتجاه نحو التغيير } \\
\hline & $.0 \mathrm{~V}$ & $\therefore 1$ & $* . r \leq 1$ & & \\
\hline & $* Y .91$ & $\cdot r \Lambda_{0}$ & $* * . .000$ & & \\
\hline & . & $\because \cdot 1$ &. .174 & & \\
\hline \multirow{4}{*}{ 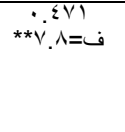 } & $\because \wedge 91-$ & $. .1 K T-$ &. .171 & عد & \multirow[t]{4}{*}{ |الاتجاه نحو تمكين المراة } \\
\hline & **Y.TV. & $\because \leqslant 01$ & $* * .7 .0$ & & \\
\hline & $\cdot \leq \varepsilon \leq$ & •.人 & $* * .0 \leq \Lambda$ & & \\
\hline & 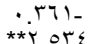 & $\because \varepsilon Y_{-}$ & $\begin{array}{l}\because 110 \\
* * \quad<9\end{array}$ & & \\
\hline \multirow{6}{*}{ 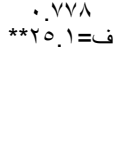 } & 1.911 & $\because 1 \cdots$ & $\star * \cdot \varepsilon \cdot \Gamma$ & |عدد السكان & \multirow{6}{*}{ مستوى الرخاء الاقتصنادي } \\
\hline & $1.91 \leq$ & 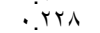 & **. .VT. & & \\
\hline & $* *$ *... & .879 & $* * \cdot . \wedge \cdot r$ & & \\
\hline & . & $\because 7 \leq$ & *.TI & & \\
\hline & 1.171 & .119 & $* * .09 \mathrm{~V}$ & الالتجاه نحو & \\
\hline & $* * Y .799$ & - & $* * . .794$ & الاتجاه نحو تمكين المر أة & \\
\hline \multirow{5}{*}{$\begin{array}{l}\because Y T T \\
i . \Lambda=0\end{array}$} & $1 . \Gamma \cdot \hat{N}_{-}$ &..$K T V_{-}$ & $\because 1 \cdots$ & عدد السكان & \multirow[t]{5}{*}{ الشعور بالعدالة الاجتماعية } \\
\hline & $.19 \cdot-$ & $\because \leq \varepsilon \leq-$ & $* . r 91$ & & \\
\hline & $\cdot \leqslant r_{0}$ & .11 & **. & & \\
\hline & & $\because .04$ & $\because 1100$ & & \\
\hline & $1 . \leqslant 90$ & $\because r_{\text {q. }}$ & **. . & | الاتجاه ذ & \\
\hline
\end{tabular}

$\Lambda Y \wedge \varepsilon$ 
Salama, F. A. and A. A. M. H. Ecresh

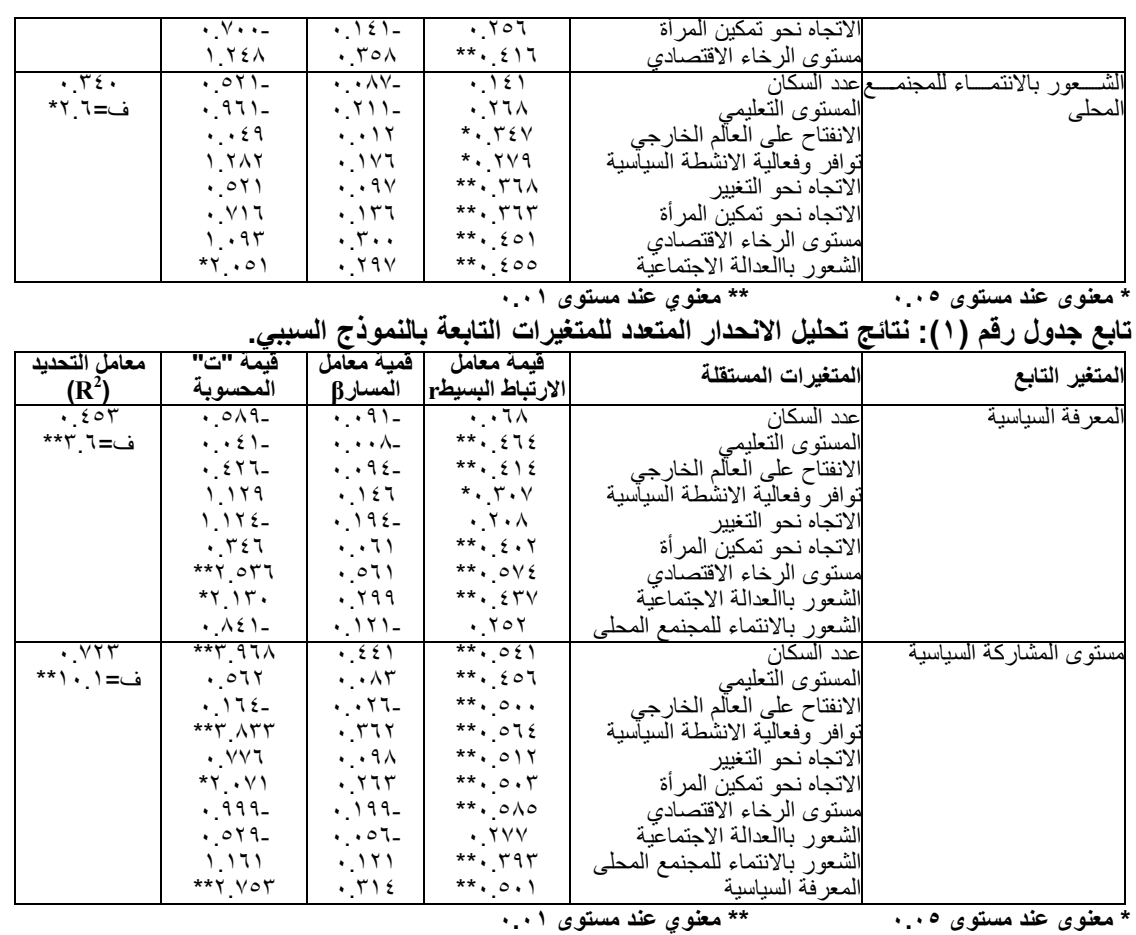

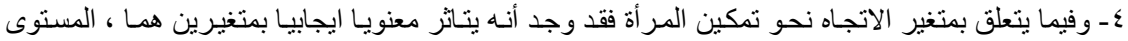

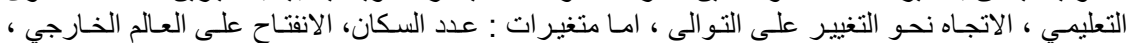

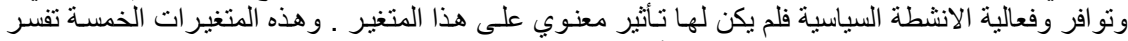

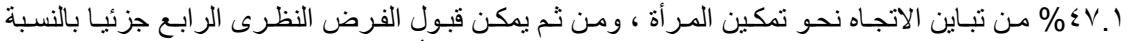

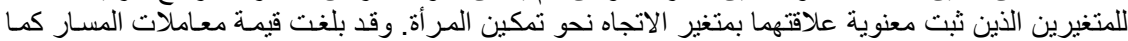

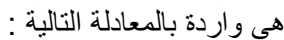
$\mathrm{X}_{6}=12.52-0.123 \mathrm{X}_{1}+0.451 \mathrm{X}_{2}+0.083 \mathrm{X}_{3}-0.042 \mathrm{X}_{4}+0.367 \mathrm{X}_{5}+\mathrm{P}_{6 \mathrm{u}} \mathrm{R}_{\mathrm{u}}$

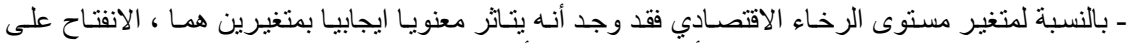

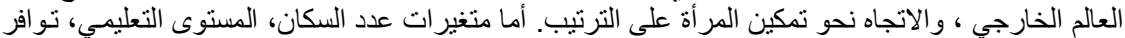

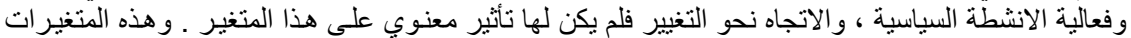

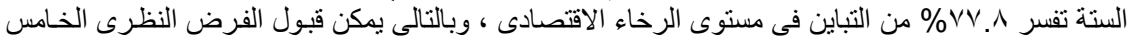

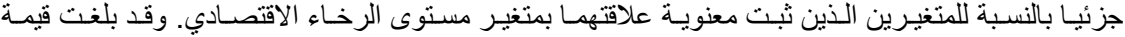
معاملات المسار كما هي واردة بالمعادلة التالية : $\mathrm{X}_{7}=154.317+0.1 \mathrm{X}_{1}+0.228 \mathrm{X}_{2}+0.369 \mathrm{X}_{3}+0.064 \mathrm{X}_{4}+0.119 \mathrm{X}_{5}+0.266 \mathrm{X}_{6}+\mathrm{P}_{7 \mathrm{v}} \mathrm{R}_{\mathrm{v}}$

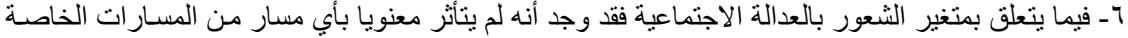

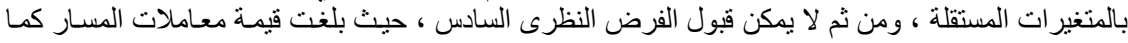

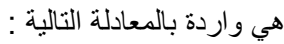
$\mathrm{X}_{8}=-6.768-0.227 \mathrm{X}_{1}-0.044 \mathrm{X}_{2}+0.11 \mathrm{X}_{3}+0.053 \mathrm{X}_{4}+0.29 \mathrm{X}_{5}-0.141 \mathrm{X}_{6}+0.358 \mathrm{X}_{7}$ $+\mathrm{P}_{8 \mathrm{v}} \mathrm{R}_{\mathrm{x}}$

وربما يرجع عدم معنوية هذه المسار ات لوجود متغير ات اخرى تؤثر فى درجة الثـعور بالعدالة

الاجتماعية ولم ترد بالنموذج المقترح عدورية 


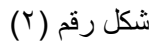

V- بالنسبة لمتغير الثعور بالانتماء للمجتمع المحلى فقد وجد أنه يتاثر معنويا ايجابيا

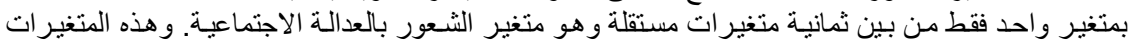

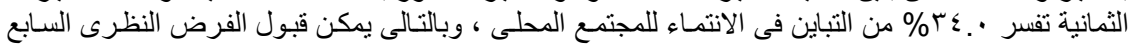

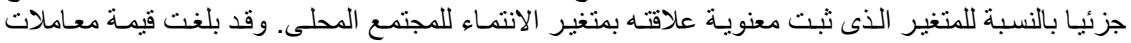
المسار كما هي واردة بالمعادلة التالية: $\mathrm{X}_{9}=6.601-0.087 \mathrm{X}_{1}-0.211 \mathrm{X}_{2}+0.12 \mathrm{X}_{3}+0.176 \mathrm{X}_{4}+0.097 \mathrm{X}_{5}+0.136 \mathrm{X}_{6}+0.3 \mathrm{X}_{7}+$ $0.297 \mathrm{X}_{8}+\mathrm{P}_{9 \mathrm{w}} \mathrm{R}_{\mathrm{W}}$

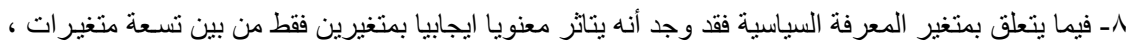

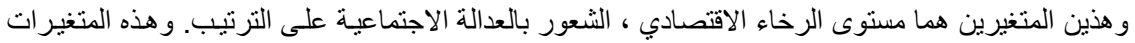

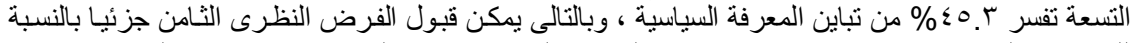

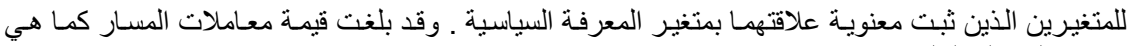
$\mathrm{X}_{10}=-1.18-0.091 \mathrm{X}_{1}-0.008 \mathrm{X}_{2}-0.094 \mathrm{X}_{3}+0.146 \mathrm{X}_{4}-0.194 \mathrm{X}_{5}+0.06 \mathrm{X}_{6}+$ $0.561 \mathrm{X}_{7}+0.299 \mathrm{X}_{8}-0.121 \mathrm{X}_{9}+\mathrm{P}_{10 \mathrm{y}} \mathrm{R}_{\mathrm{y}}$

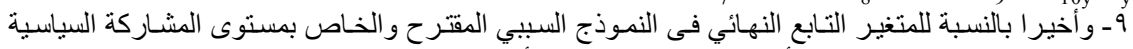

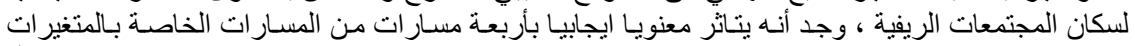

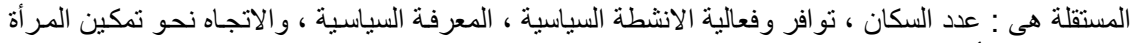

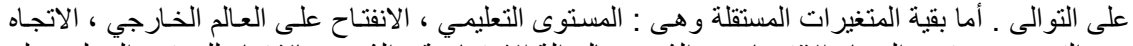

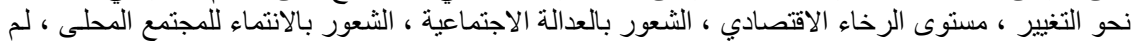

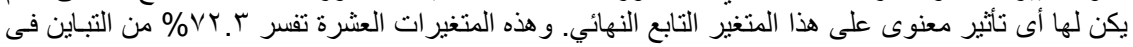

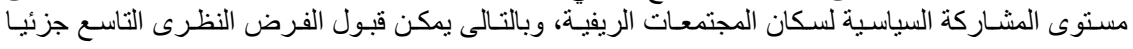


Salama, F. A. and A. A. M. H. Ecresh

بالنسبة للمتغير ات الذى ثبت معنوية علاقتها بمتغير المشاركة السياسية ـ وقد بلغت قيمة معاملات المسار كما هي واردة بالمعادلة النالية:

$X_{11}=-4.538+3.968 X_{1}+0.562 X_{2}-1.64 X_{3}+3.833 X_{4}+0.776 X_{5}+2.071 X_{6}-0.999$ $\mathrm{X}_{7}-0.529 \mathrm{X}_{8}+1.161 \mathrm{X}_{9}+2.753 \mathrm{X}_{10}+\mathrm{P}_{11 \mathrm{z}} \mathrm{R}_{\mathrm{z}}$

ويبين الثكل رقم (r) النالي النموذج السببي المعدل لمستوى المشاركة السياسية لسكان المجتمعات

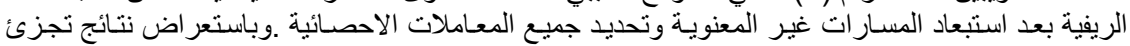

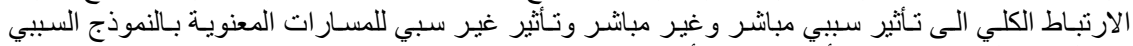

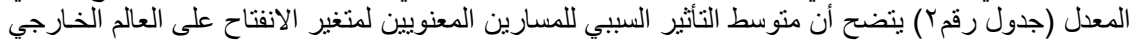

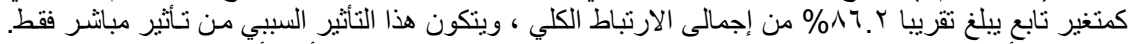

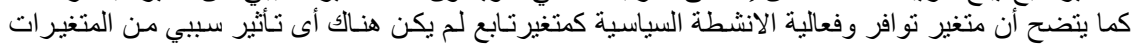

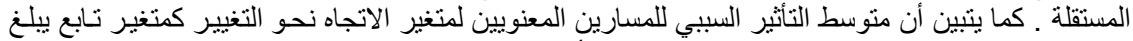

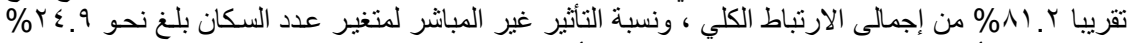

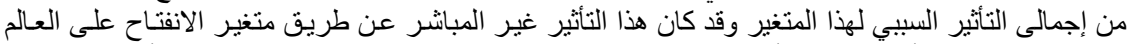

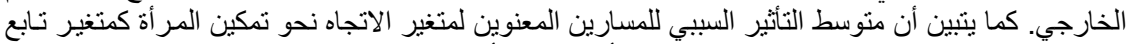

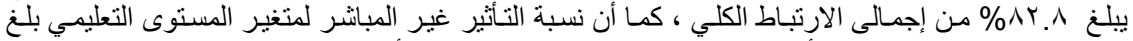

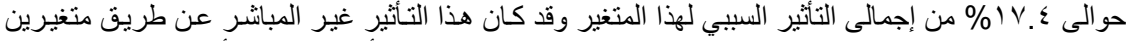

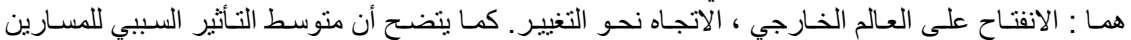

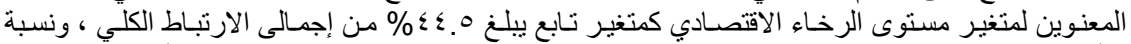

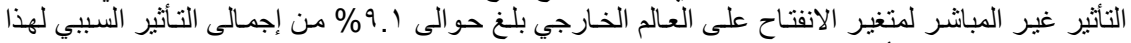

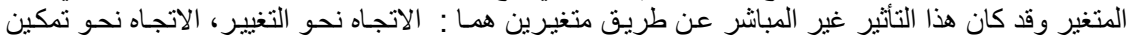

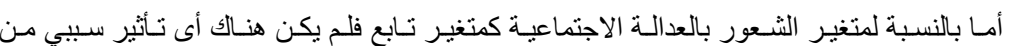
المرأة .

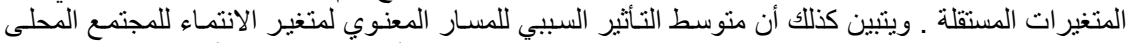

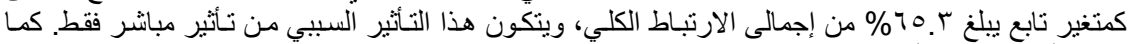

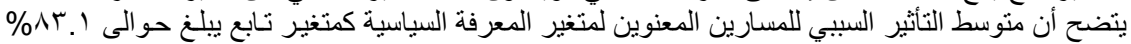

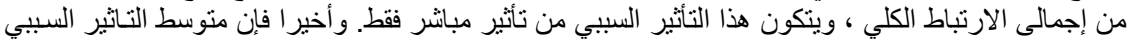

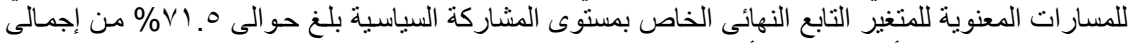

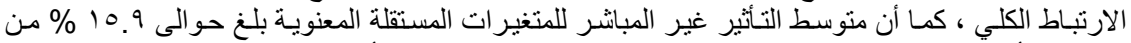

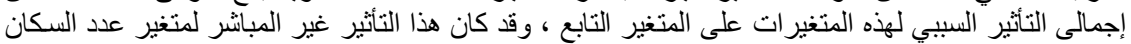

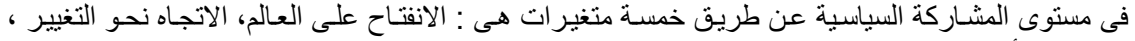

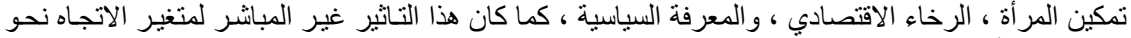

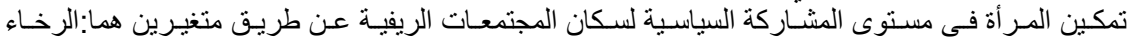

الاقتصادي، والمعرفة أفى السياسية.

جدول رقم (Y): التأثثر المباشر وغير المباشر والارتباط غير السببي للمسارات المعنويـة بالنموذج السببيب المعدل . المبالكر المبر

\begin{tabular}{|c|c|c|c|c|c|c|c|}
\hline \multirow{2}{*}{ الارتباطي } & \multirow{2}{*}{ التيري } & \multirow{2}{*}{ السببي } & \multicolumn{2}{|l|}{ التأثير غير المباشر } & \multirow{2}{*}{ 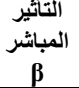 } & \multirow{2}{*}{ |المتغيرات المستقلة } & \multirow[b]{2}{*}{ |المتغير التابع } \\
\hline & & & عن طريق & قيمته & & & \\
\hline$\cdot . \$ 19$ & $\because 9$. & •rrq & -- & -- & - זrq & إعلد السكان & الانفتـاح على العـالد \\
\hline$\therefore .11$ & $\because \leq \varepsilon$ & $\therefore .7 V \varepsilon$ & -- & -- & $\cdot .7 \vee \varepsilon$ & ||لمستوى التعليمي & الخارجي \\
\hline--- & --- & --- & -- & -- & --- & & 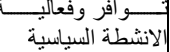 \\
\hline $.0 \leqslant V$ & $\because$ ? & .0 .9 & لانفتاح على العالم الخارجي &. $.1 T \mathrm{~V}$ & $\cdot$. TNY & |عدد السكان & |الاتجاه نحو التغيير \\
\hline .000 & $\therefore \mathrm{V}$. & $\therefore$ r 100 & -- & -- & $\cdot r 10$ & |الانفتاح على العالم الخارجي & \\
\hline .7 .0 & .90 & $.0 \leqslant 7$ & الانفتاح على العالم،الاتجاه & .990 & . $\leqslant 01$ & & الاتجــاه نحــو تمكـين \\
\hline$\cdot .295$ & דואו & $\therefore$ rty & نحو التغيير & $\cdot$ & $\therefore$ TrV & |الاتجاه نحو التفيير & |المرأة \\
\hline$\because A \cdot r$ & •. ז94 & $\cdot \varepsilon \cdot 4$ & الاتجاه نحو التغيير، تمكين & $\therefore \mathrm{rV}$ & $\cdot .499$ & ،|الانفتاح على العالم الخارجي & ى الرخــاء \\
\hline .794 & $\therefore$ ¿हY & $\therefore$. Y Y4 & المرأة & -- & $\cdot .174$ & |الاتجاه نحو تمكين المرأة & لاقتصادب \\
\hline
\end{tabular}

$\wedge \mathrm{\wedge} \vee$ 
J. Agric. Sci. Mansoura Univ., 33(11), November, 2008

\begin{tabular}{|c|c|c|c|c|c|c|c|}
\hline & & & -- & & & & \\
\hline-- & -- & -- & -- & -- & -- & & 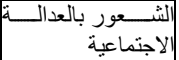 \\
\hline$\because \leqslant 00$ & .101 & $\because$ rqV & -- & -- & $\because$ Y & الثعور بالعدالة الاجتماعية & 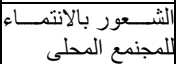 \\
\hline $.0 V \leqslant$ &.$\cdot 1 \pi$ & .071 & -- & - & .071 & مستوى الرخاء الاقتتصادي & |المعرفة السياسية | \\
\hline$\cdot . \varepsilon r v$ &. $.1 \mathrm{\mu}$ &..$Y 99$ & -- & -- & $\cdot .499$ & الثعور بالعدالة الاجتمـاعية & \\
\hline $.0 \leqslant 1$ & $\because \cdot 1 r$ & $\because 049$ & 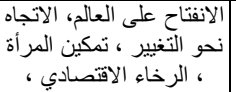 & $\because \cdot \wedge \wedge$ & $\because \varepsilon \leqslant 1$ & عدد السكان ع ع ع & |المشاركة السياسية \\
\hline $.07 \leqslant$ & $\cdot r \cdot r$ & (זדת & المعرفة السباسية & -- & 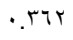 & تـــــوافر وفعاليــــة الانشـــــة & \\
\hline $.0 . \mu$ & .194 & . & -- & $\because \varepsilon V$ & שדציט & |لاسيسية | & \\
\hline .0 .1 & $\because 1 \wedge \mathrm{V}$ & $\cdot M \leq$ & 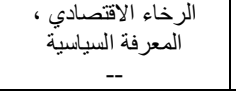 & -- &.$\mu$ & المعرفة السياسية تحكين المرأة & \\
\hline
\end{tabular}

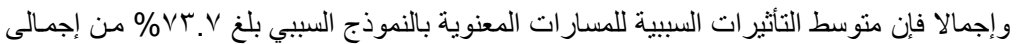

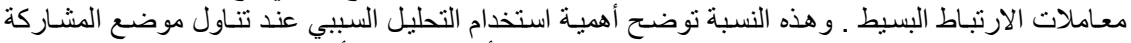

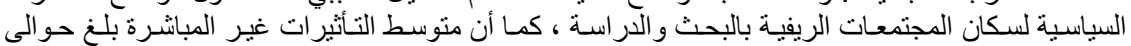

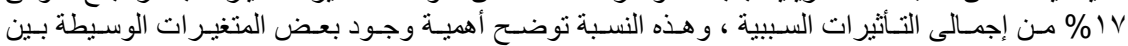

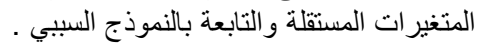

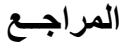

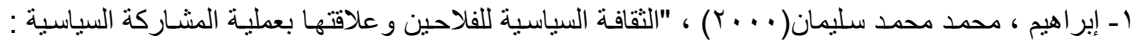

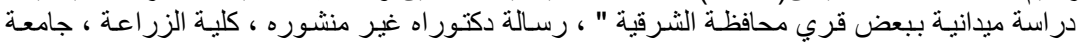

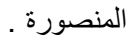

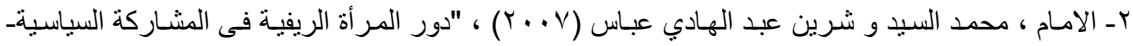

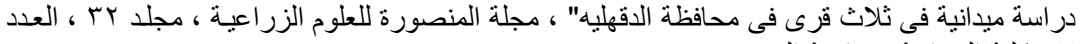

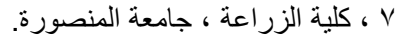

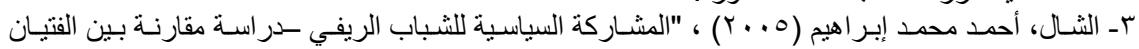

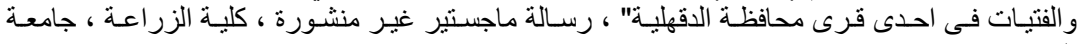
المنصورة .

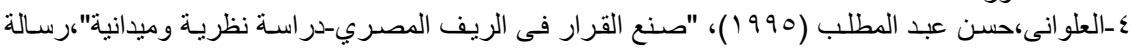

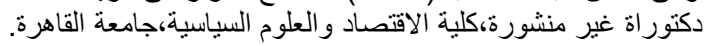

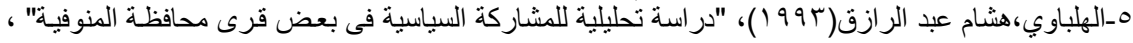

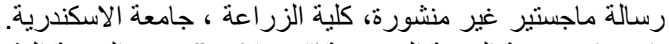

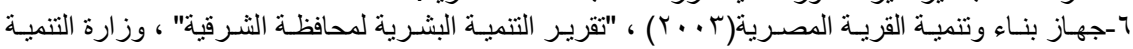
المحلية ، مصر.

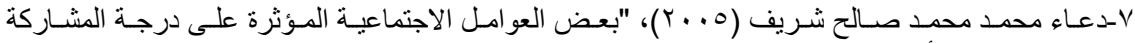

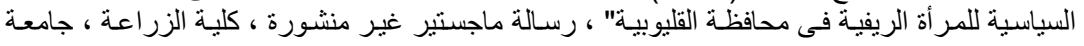

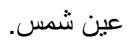

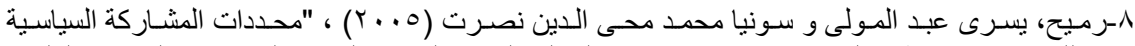

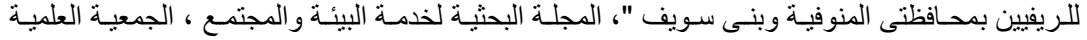
لحماية البيئة الريفية بالشرقية ، مجلد 1 ، عدد ال، ، الزقازيق، محافظة الثرقية. 


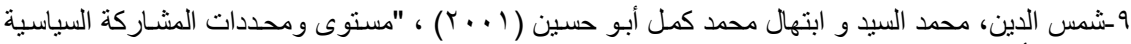

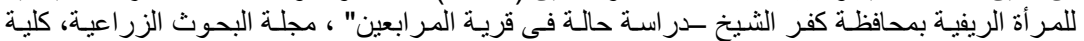

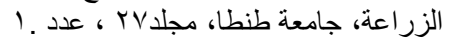

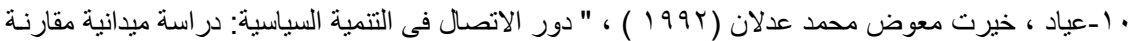

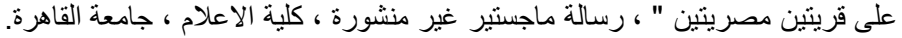

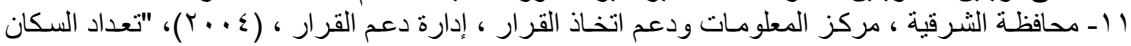
التقديري .

12-Alwin , Duane F. and Robert M. Hauser. (1975). The Decomposition of Effect in Path Analysis , American Sociological Review 40 :37-47.

13-Asher, Herbert B. (1976) . Causal Modeling, Sag Publication , Inc., U.S.A.

14-Conway, M. (1985). Political Participation in the United States, Washington, DC: Congressional Quarterly, Inc

15-Cornwall ,Andrea and Karen Brock .2005. "Beyond Buzzwords : Poverty Reduction, Participation and Empowerment in Development Policy", United Nations Research Institute for Social Development, Overarching Concerns Programme Paper Number 10, November 2005.

16-Dalton , Russell J..(2008). "Citizenship Norms and the Expansion of Political Participation", POLITICAL STUDIES, VOL 56, 76-98.

17-Jr., William P. Eveland.;; Hayes , Andrew F.; Shah, Dhavan V. and Nojin Kwak.(2005). "Understanding the Relationship Between Communication and Political Knowledge: A Model Comparison Approach Using Panel Data", Political Communication, 22:423-446.

18-Pindare , Paul.(1984)."Socio-Economic Development and Political Participation in Borono State of Nigeria" , in D.M..I., 44, No.11.

19-Verba S.,Nie and Jae-on kim," Participation and Political Equality" , Cambridge: Cambridge Unv. Press,1978

20-Weber , Lori .(2003) "Rugged individuals and social butterflies: the consequences of social and individual political participation for political tolerance", The Social Science Journal 40 , Issue 2 , 335342.

\section{A PATH ANALYSIS OF SOME COMMUNITY FACTORS AFFECTING ON POLITICAL PARTICIPATION OF THE INHABITANTS OF RURAL COMMUNITIES IN SHARKIA GOVERNORATE-EGYPT \\ Salama, F. A. ${ }^{*}$ and A. A. M. H. Ecresh ${ }^{* *}$ \\ * Faculty of Agriculture - Minufiya University \\ ** Faculty of Agriculture - Zagazig University}

\section{ABSTRACT}

The primary objective of this study was to develop and test the importance of a set of factors that are posited as explaining the level of political participation of inhabitants of rural communities.

A purposive sample of 50 villages in $999999 \%$ Sharkia governorate was carried out . A sample of 150 informants was chosen by a Sociometric method to 


\section{J. Agric. Sci. Mansoura Univ., 33(11), November, 2008}

collect the data which were personally interviewed through a questionnaire and which were analyzed by path analysis technique.

The findings indicated that openness on the outside world $\left(R^{2}=0.621\right)$ affected by size of population and educational level, availability and effectiveness of political activities affected by no variable, the attitude towards change $\left(R^{2}=0.428\right)$ affected by openness to the outside world and size of population, the attitude towards women empowerment $\left(R^{2}=0.471\right)$ affected by educational level and the attitude towards change, the level of economic prosperity $\left(R^{2}=0.778\right)$ affected by openness to the outside world and the attitude towards women empowerment, sense of social justice affected by no variable, sense of belonging to the community $\left(R^{2}=0.34\right)$ affected by sense of social justice, political knowledge $\left(R^{2}=0.453\right)$ affected by the level of economic prosperity and sense of social justice, the level of political participation $\left(R^{2}=0.723\right)$ affected by size of population, availability and effectiveness of political activities, political knowledge and the attitude towards women empowerment. Finally, a decomposition of simple correlation into their components was made for the significant path in the revised model to show the importance of the casual analysis and the intervening variables as well. 